\title{
Phase Control of Self-Excited Parametric Resonators
}

\author{
James M.L. Miller, ${ }^{1, *}$ Dongsuk D. Shin, ${ }^{1}$ Hyun-Keun Kwon, ${ }^{1}$ Steven W. Shaw, ${ }^{2,3}$ and \\ Thomas W. Kenny ${ }^{1}$ \\ ${ }^{1}$ Departments of Mechanical and Electrical Engineering, Stanford University, Stanford, California 94305, USA \\ ${ }^{2}$ Department of Mechanical and Aerospace Engineering, Florida Institute of Technology, Melbourne, \\ Florida 32901, USA \\ ${ }^{3}$ Departments of Mechanical Engineering and Physics and Astronomy, Michigan State University, East Lansing, \\ Michigan 48823, USA
}

(Received 31 May 2019; revised manuscript received 26 July 2019; published 23 October 2019)

The phase state of a parametric resonator provides important information about the nonlinear damping and the ability to employ feedback to achieve self-sustained oscillations. We derive the minimum nonlinear damping value required to enable phase control of Duffing parametric resonators and confirm this using tunable nonlinear micromechanical devices with nonlinear damping values below and above the critical value. For softening resonators with nonlinear damping values beyond the critical value, we demonstrate parametric phase control to experimentally operate on the unstable branches of the parametric response. Parametric phase locking is an alternate method for forming a timing reference from a micromechanical resonator, provides a sensitive technique for measuring the nonlinear parameters, and enables future closed-loop measurements of the dynamics of driven single and coupled parametric resonators.

DOI: 10.1103/PhysRevApplied.12.044053

\section{INTRODUCTION}

Parametric resonance is a common phenomenon in driven high-quality-factor $(Q)$ vibrational systems, arising in optical [1-3], microwave [4-6], and mechanical modes [7-10]. Modulation of a reactive parameter in a resonant system, such as the stiffness of a mechanical mode or the inductance of a microwave cavity, near twice the resonant frequency can parametrically amplify the system. If this frequency modulation exceeds the parametric instability threshold, period-two oscillations will commence at one of two distinct parametric phase states [11]. These two states have equal amplitude and differ only by a time-translation shift of $\pi$ radians. The two-state dynamics of classical and quantum parametric resonators have been an area of intense interest, with ongoing studies of fluctuation-activated interstate switching [12-14], broken time-translation symmetry [15,16], time crystals [17], and parametric logic operations [18-23].

Beyond the parametric instability threshold, the Duffing nonlinearity and nonlinear damping control the response

*jmlm@stanford.edu

Published by the American Physical Society under the terms of the Creative Commons Attribution 4.0 International license. Further distribution of this work must maintain attribution to the author(s) and the published article's title, journal citation, and DOI. and their relative effects result in rich dynamical behavior [11,24-27]. Micro- and nanoelectromechanical (MEM and NEM) resonators provide an ideal platform for experimental study of the dynamics of nonlinear parametric resonators, because of the easily accessible nonlinear ceiling and the ability to tune the nonlinearities via the device architecture and the local electric field profile. They also present numerous applications for parametric pumping in, for example, scanning probe microscopes [7-9], signal amplifiers [15,28,29], and inertial sensors [30-32] and for generating nonclassical states of motion [7,33-38].

The dynamics of a single phase state in a parametric resonator have a nontrivial dependence on the relative Duffing nonlinearity and nonlinear damping and have received little attention $[14,16,39]$. In parametric resonators exhibiting simultaneous Duffing nonlinearity and nonlinear damping, the phase-frequency curves spontaneously flip sign when the nonlinear damping exceeds a critical value. This suggests a phase-control-based method for operating a parametric resonator.

MEM and NEM resonators often serve as the high$Q$ frequency-determining elements in resonant sensors and timing references [40,41]. The conventional oscillator topology implements direct feedback [42-47], which involves some form of external or internal velocityproportional feedback at the resonant frequency [10]. Alternately, the phase lag between the displacement and forcing of a mode can be leveraged to construct a direct 
phase-locked loop (PLL), which has the advantage of not requiring a thermomechanical noise-limited readout $[48,49]$. Recently, parametric resonance has been explored as an autonomous oscillator topology; by using feedback that modulates the spring constant of a mode at twice the resonant frequency, parametric oscillations will occur with potentially better phase noise than conventional direct feedback oscillators [50].

In this paper, we demonstrate a fourth technique for creating a timing reference from a mode: a parametric phase-locked loop. A parametric PLL can access the unstable parametric resonance response and is a viable experimental technique for stable operation on all five periodic responses of a driven parametric resonator exhibiting broken time-translation symmetry [16]. A parametric PLL can be utilized if the nonlinear damping in the mode exceeds a critical value. We theoretically delineate the phase dynamics in the vicinity of this critical nonlinear damping value and verify the dynamics using micromechanical cantilevers with tunable nonlinearities. Direct phase control is routinely used to access the unstable periodic response of directly driven nonlinear resonators, where no discontinuity exists between the stable and unstable branches [5153], and can be modified into a "trajectory-locked loop" configuration for measuring the bifurcation frequencies in the nonlinear regime [54]. Many previously demonstrated parametric resonators have insufficient intrinsic nonlinear damping to stabilize the response without introducing artificial nonlinearities into the feedback $[50,55]$. We demonstrate devices with sufficient intrinsic nonlinear dissipation to automatically stabilize the phase-controlled parametric response [45].

\section{OPEN-LOOP PARAMETRIC RESONANCE}

\section{A. Model}

The displacement, $x$, of a parametrically pumped nonlinear resonator is given by

$$
\ddot{x}+\gamma \dot{x}+\omega_{0}^{2} x+\alpha x^{3}+\eta x^{2} \dot{x}+\lambda \cos (2 \omega t) x=0,
$$

where $\omega_{0}$ is the natural frequency, $\gamma=\omega_{0} / Q$ is the linewidth, $Q$ is the quality factor, and $\lambda$ is the parametric pump strength. Equation (1) includes two nonlinearities: the nonlinear damping parameter, $\eta$, and the Duffing parameter, $\alpha$. We ignore the thermal additive noise that accompanies the linear damping and the frequency noise that accompanies the nonlinear damping [56]. For a parametric pump at $2 \omega=2\left(\omega_{0}+\sigma\right)$, where the frequency detuning $\sigma \ll \omega_{0}$, the effective damping becomes negative when $\lambda>\lambda_{\text {thresh }}\left(\omega_{0}+\sigma\right)=2 \gamma \omega_{0} \sqrt{1+(2 \sigma / \gamma)^{2}}$, initiating parametric oscillations at an amplitude and phase governed by $\alpha$ and $\eta$.

The frequency-controlled parametric resonance dynamics can be predicted from Eq. (1) using perturbation theory.
This corresponds to an applied parametric pump beyond threshold at some frequency, $2 \omega$, which causes the device to self-oscillate with some amplitude, $r$, and phase lag, $\phi$, behind the pump. The method of averaging can be employed to solve Eq. (1) for the slowly varying amplitude and phase of motion $[15,57,58]$. This solution strategy begins by assuming solutions of the form

$$
x(t)=r \cos (\omega t+\phi), \quad \dot{x}(t)=-r \omega \sin (\omega t+\phi),
$$

which must satisfy the following equation of constraint:

$$
0=\dot{r} \cos (\omega t+\phi)-r \dot{\phi} \sin (\omega t+\phi) .
$$

We substitute Eq. (2) and its derivatives into Eq. (1), impose the constraint in Eq. (3), then average over one cycle of vibrations while assuming that $r$ and $\phi$ do not appreciably change over a single period. This yields two differential equations for the amplitude, $r$, and phase, $\phi$ :

$$
\begin{aligned}
& \dot{r}=-\frac{\gamma r}{2}-\frac{\eta r^{3}}{8}+\frac{\lambda r \sin (2 \phi)}{4 \omega}, \\
& \dot{\phi}=\frac{\left(\omega_{0}^{2}-\omega^{2}\right)}{2 \omega}+\frac{3 \alpha r^{2}}{8 \omega}+\frac{\lambda \cos (2 \phi)}{4 \omega},
\end{aligned}
$$

where at $r=0, \phi$ is not defined; this situation is not of interest in the present work. Setting Eqs. (4) and (5) to zero provides steady-state conditions for the amplitude and phase. The trivial response always exists, but for $\lambda>\lambda_{\text {thresh }}$, nontrivial responses may occur and can be conveniently expressed by the following:

$$
\begin{aligned}
r_{ \pm} & =\sqrt{\frac{2\left\{2 \gamma\left(\omega_{0}+\sigma\right)+c_{ \pm}\left[\lambda+2 \sigma\left(2 \omega_{0}+\sigma\right)\right]\right\}}{3 \alpha c_{ \pm}-\eta\left(\omega_{0}+\sigma\right)},} \\
\phi_{ \pm} & =-\arctan \left(c_{ \pm}\right), \\
c_{ \pm} & =\frac{-3 \lambda \alpha \pm \sqrt{(3 \lambda \alpha)^{2}-\left(\omega_{0}+\sigma\right)^{2} \mathfrak{C}_{+} \mathfrak{C}_{-}}}{\left(\omega_{0}+\sigma\right) \mathfrak{C}_{+}} \\
\mathfrak{C}_{ \pm} & =6 \alpha \gamma \pm \eta\left[\lambda \pm 2 \sigma\left(2 \omega_{0}+\sigma\right)\right] .
\end{aligned}
$$

Figure 1 depicts the amplitude-frequency and phasefrequency curves from Eqs. (6) and (7), respectively, for a parametric resonator with values of $\gamma, \omega_{0}, \alpha$, and $\eta$ attainable using a micromechanical platform. The stable branch of the amplitude and phase solutions in Eqs. (6) and (7) corresponds to the negative root in Eqs. (8) and (9), while the unstable branch corresponds to the positive root. Figures 1(a) and 1(b) present the solutions for zero nonlinear damping. The response is characterized by a stable branch that theoretically extends out to infinite amplitude, never merging with the unstable branch, and has a positive phase slope for all frequencies, unlike the phase slope of 




(b)
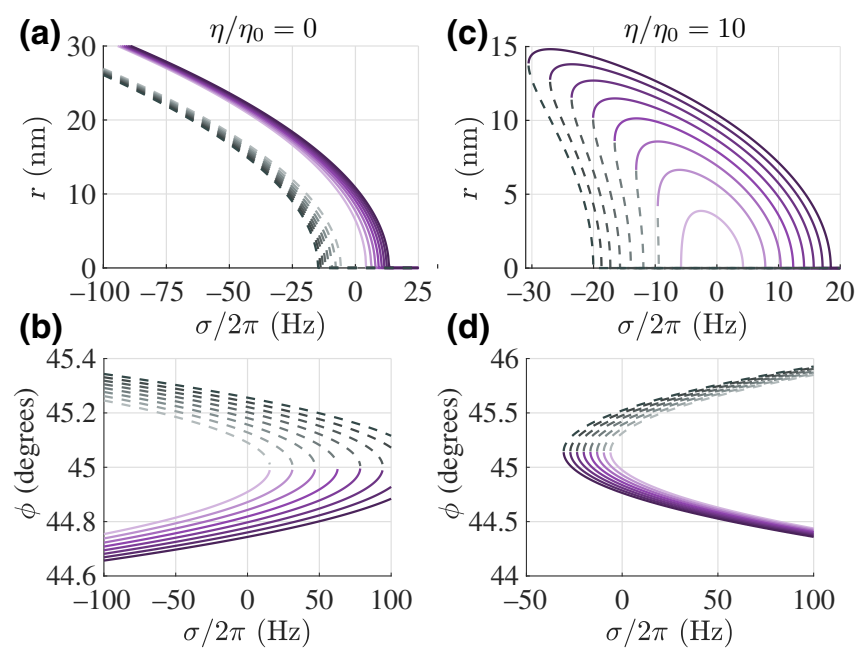

FIG. 1. The dynamics of a softening parametric resonator in the vicinity of the critical nonlinear damping, $\eta_{0}$. The (a),(c) amplitude-frequency and (b),(d) phase-frequency curves from Eqs. (6) and (7), respectively, shown for a parametric resonator with a resonant frequency of $\omega_{0}=2 \pi \times 500 \mathrm{kHz}$, a quality factor of $Q=1000$, a Duffing parameter of $\alpha=$ $-10^{24} \mathrm{rad}^{2} \mathrm{~m}^{-2} \mathrm{~s}^{-2}$, and a nonlinear damping of (a),(b) $\eta=$ $0 \mathrm{rad} \mathrm{m}^{-2} \mathrm{~s}^{-1}$, and (c),(d) $\eta=4.78 \times 10^{15} \mathrm{rad} \mathrm{m}^{-2} \mathrm{~s}^{-1} \cdot \sigma=\omega-$ $\omega_{0}$ is the frequency detuning. The normalized pump amplitude ranges from $\lambda / \lambda_{\text {thresh }}-1=5 \times 10^{-6}$ to $4 \times 10^{-5}$ in (a),(b) and $7.5 \times 10^{-5}$ in (c),(d) (light to dark curves).

a directly driven mode in its linear regime [59]. In practice, the device pulls into a side electrode at sufficiently large amplitudes [57]. As $\eta$ is increased, the phase slope decreases, as is shown in Fig. 2, until $\eta$ crosses a critical value [57]:

$$
\eta_{0}=-\frac{3 \alpha \gamma}{2 \omega_{0}^{2}} .
$$

For $\eta>\eta_{0}$, the phase slope becomes negative and the stable and unstable branches merge. Figures 1(c) and 1(d) depict the solutions with nonlinear damping well in excess of the critical value. In this regime, the phase slope is negative and parametric phase control is possible. This relationship holds for the hardening case $(\alpha>0)$ as well, where the stable-branch phase slope is negative unless the (negative) nonlinear damping is decreased below the critical value.

MEM and NEM resonators provide an excellent platform for confirming these dynamics because beams and membranes exhibit intrinsic nonlinear damping and electric fields can be applied to tune $\alpha$. Parametric resonance has been demonstrated in many devices with $\eta<\eta_{0}$ ([12, $13,19,58,60-62])$ as well as $\eta>\eta_{0}([63,64])$. We tune the intrinsic nonlinear damping and Duffing nonlinearity in MEM resonators to observe both regimes and demonstrate closed-loop stabilization of the unstable branch.
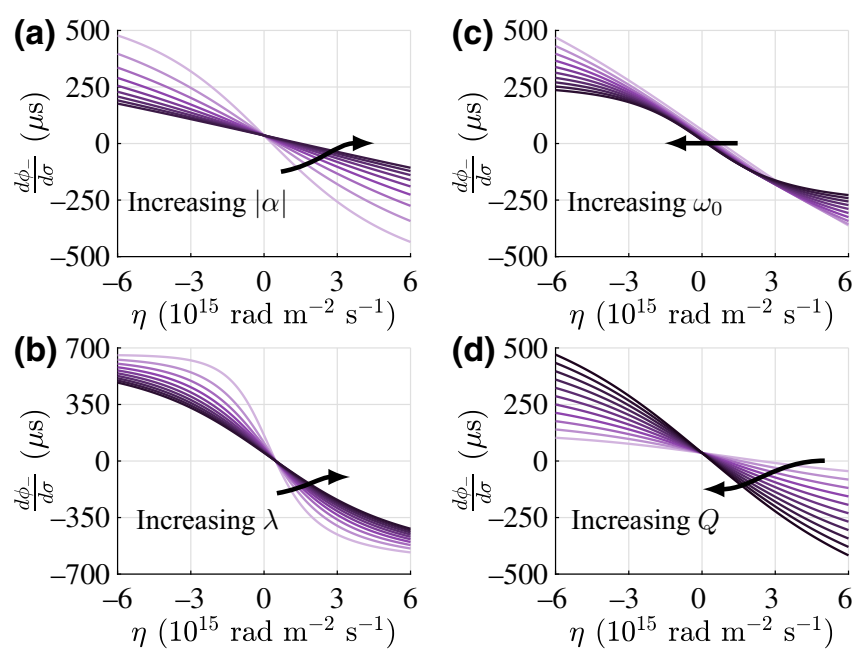

FIG. 2. The dependence of the phase slope of the stable branch, $d \phi_{-} / d \sigma$, on the nonlinear damping, $\eta$, for increasing (a) Duffing parameter, $\alpha<0$, (b) pump strength, $\lambda$, (c) natural frequency, $\omega_{0}$, and (d) quality factor, $Q$. The intersections of the curves with $d \phi_{-} / d \sigma=0$ define $\eta_{0}$. (a) $\alpha$ varying from $-7 \times 10^{23}$ to $-3.1 \times 10^{24} \mathrm{rad}^{2} \mathrm{~m}^{-2},(\mathrm{~b})\left(\lambda / \lambda_{\text {thresh }}-1\right)$ varying from $2 \times 10^{-6}$ to $2 \times 10^{-5}$, (c) $\omega_{0} / 2 \pi$ varying from $300 \mathrm{kHz}$ to $1.2 \mathrm{MHz}$, and (d) $Q$ varying from 200 to 1200 . The constant parameters are set to $\alpha=-1 \times 10^{24} \operatorname{rad}^{2} \mathrm{~m}^{-2}, \lambda / \lambda_{\text {thresh }}-1=4 \times 10^{-5}$, $\omega_{0} / 2 \pi=500 \mathrm{kHz}$, and $Q=1000$.

\section{B. Devices and measurements}

Figure 3 depicts the device and the measurement setup. The geometry is an in-plane cantilevered structure, with the base split into a wide "spring" beam and a narrow "sense" beam to enable piezoresistive sensing of the motion $[65,66]$. The resonators are fabricated in a waferscale encapsulation process, which produces ultrastable MEM devices in a hermetic vacuum-sealed environment [67]. The resonators and measurement amplifiers are tested in a temperature-controlled oven at $25^{\circ} \mathrm{C}$. Application of a voltage to an electrode adjacent to the cantilever shifts the resonant frequency of the fundamental in-plane mode, $\omega_{0}$,

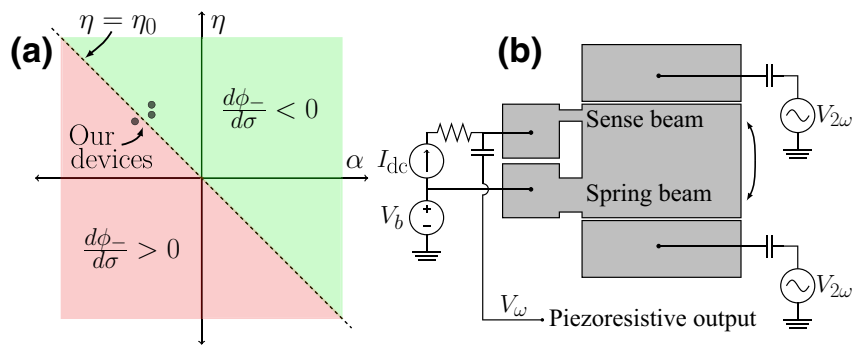

FIG. 3. Operation in the vicinity of the critical nonlinear damping. (a) The regimes of positive and negative stable-branch phase slope in the $\alpha-\eta$ plane. (b) The measurement schematic for our microcantilevered parametric resonators with tunable nonlinearities. 
via electrostatic softening [26]. Variation of this voltage near $2 \omega_{0}$ modulates the stiffness, parametrically pumping the flexural mode. By modifying the length of the supporting beams and the gap size between the cantilever and the electrodes, the relative influence of the electrostatic Duffing parameter and the nonlinear damping on the motion can be tuned. The electrostatic component of the Duffing nonlinearity, $\alpha_{e}$, dominates because of the large bias voltages, $V_{b}$, as well as the large electrode area, $A$, on either side of the device [68]:

$$
\alpha_{e}=-\frac{8 \epsilon_{0} A V_{b}^{2}}{m g^{5}}
$$

where $g$ is the gap width between the resonator and the adjacent electrodes and $\epsilon_{0}$ is the vacuum permittivity. The nonlinear damping is believed to arise from tension in the sense beam and can be increased by reducing its length. Analogous nonlinear damping has been observed in doubly clamped beams [45,69-72], guitar strings [16], carbon nanotubes [73], and graphene [64,73-76].

Figure 4 plots the measured frequency-controlled parametric resonance sweeps for devices with $\eta<\eta_{0}$ and $\eta>$ $\eta_{0}$, which confirms the change in sign of the phase slope in Fig. 1. We apply a bias voltage of $120 \mathrm{~V}$ and sweep a parametric pump across twice the resonant frequency using both electrodes adjacent to the device, while using a lockin amplifier to demodulate the output of the piezoresistive readout to obtain the amplitude and phase of the signal at one half the pump frequency. The device is equally likely to initiate parametric vibrations at either of two phases, separated by $\pi$ radians. The phase dynamics for the two phase states are identical in the absence of a direct drive


FIG. 4. The amplitude $(r)$ and phase $(\phi)$ for reverse frequency sweeps and increasing parametric pumping, for devices with nonlinear damping below and above $\eta_{0} . \sigma=\omega-\omega_{0}$ is the frequency detuning. Device (a),(b) A (narrow gaps and long support beams) and (c),(d) C (wide gaps and short support beams).
[16], so we present measurements from only one of the states. We calibrate the voltage amplitude at the output of the piezoresistive readout to units of displacement using the thermomechanical motion $[41,57,77]$.

\section{PARAMETRIC PHASE CONTROL}

\section{A. Comparison to other techniques}

Frequency control is useful for characterizing the dynamics of the stable branches of a parametric resonator but is unable to access the unstable branches. By changing the control variable from the frequency to the phase set point, $\delta$, the entire nontrivial parametric response is accessible, provided that the stable and unstable branches join (the nonlinear damping exceeds the critical value).

A parametric PLL uses an above-threshold parametric pump at twice the resonant frequency to induce vibrations at the mechanical resonant frequency and controls the phase between the pump and displacement to operate anywhere in the nontrivial steady-state response. It is useful for characterizing the nonlinear behavior of a parametric resonator, which is the focus of this paper, and also provides an alternate method to build a timing reference from a mechanical mode. We will first briefly discuss timing reference topologies based on direct phase-locked loops, direct feedback, and parametric feedback and then explain how the parametric PLL topology is distinct from these other approaches.

A direct phase-locked loop outputs a voltage with a frequency, $\omega$, near the mechanical resonant frequency, which is applied to actuate the resonator at $\omega$. For a drive near resonance $\left(\omega \approx \omega_{0}\right)$, the resonator displacement lags $\pi / 2$ radians behind the forcing. In an analog PLL, the output signal is generated by a voltage-controlled oscillator (VCO), while a digital PLL uses a numerically controlled oscillator (NCO). The resonator motion is measured, filtered, and converted into a signal at $\omega$ that is fed into the direct PLL input. The PLL measures the phase difference between the $\omega$ signal at its output and input and compares this to the desired phase condition. If the resonator is in its linear regime, the phase slope at resonance is negative, so an increase in the frequency of the $\mathrm{VCO}$ or $\mathrm{NCO}$ will increase the phase lag of the displacement behind the forcing and vice versa. The PLL utilizes a proportionalintegral-derivative (PID) loop to adjust the frequency of the output signal to maintain vibrations at the desired phase condition. In this way, the VCO or NCO frequency is determined by the resonant frequency of the mechanical resonator, so the frequency of the PLL can be used as a timing reference.

Unlike the direct PLL, a direct feedback oscillator does not use an external oscillator to drive the resonator. Instead, the thermomechanical vibration of the resonator at the resonant frequency is measured, filtered, amplified, phase shifted, and directly applied back as an external 
velocity-proportional force. This enhances the effective quality factor of the mechanical resonance and will induce self-oscillations beyond a certain threshold. The selfoscillations will track the mechanical resonant frequency and can be used as a timing reference. Because the effective damping in a self-oscillator is negative, the vibration amplitude will be unbounded unless there is a nonlinearity to stabilize the amplitude. The nonlinearity is traditionally implemented in the feedback circuitry but it can also rely on the nonlinear response of the resonator itself [45].

A parametric feedback oscillator is analogous to a direct feedback oscillator, in that no external oscillator is required, but the feedback signals are applied at twice the resonant frequency. The resonator motion at the resonant frequency is measured, filtered, and phase shifted. The resulting signal is frequency-doubled and fed into a band-pass filter to apply a $2 \omega$ signal as a parametric pump, which modulates the resonator spring constant. With sufficient parametric feedback amplitude, the resonator will self-oscillate at the resonant frequency and this signal can be used to build a timing reference. As for a direct feedback oscillator, the resonator amplitude will grow exponentially unless a nonlinearity is imposed by the feedback electronics or by the resonator.

A parametric PLL is analogous to a direct PLL because it uses a PID loop to establish a desired phase condition between its output oscillator and the input from the resonator motion. But instead of applying a drive at $\omega$, the parametric PLL modulates the resonator spring constant at $2 \omega$, beyond the parametric resonance threshold, to induce parametric oscillations. The parametric PLL maintains a phase condition between its output signal, at $2 \omega$, and the vibrations at $\omega$. Just as for direct feedback and parametric feedback, the resonator requires a nonlinearity to stabilize the amplitude, which for our devices is due to the intrinsic nonlinearity. A parametric PLL is different from a parametric feedback oscillator because the measured vibrations are not fed back to parametrically pump the motion. The signal from the parametric resonator is only used to compute the phase difference with the VCO or NCO, so the only noise that goes into the pump channel comes from the VCO or NCO.

\section{B. Model}

We model the parametric phase-controlled dynamics by modifying Eq. (1) as follows:

$$
\begin{aligned}
0= & \ddot{x}+\gamma \dot{x}+\omega_{1}^{2} x+\alpha x^{3}+\eta x^{2} \dot{x} \\
& +\lambda \cos \left[2\left(\omega_{1} t+\phi\right)+\delta\right] x,
\end{aligned}
$$

where we introduce a shifted resonant frequency, $\omega_{1}$, to account for the pump-induced dc electrostatic softening [57]. $\delta$ is the phase set point of the parametric phase-locked loop. Equation (12) permits solutions of the following form:

$$
x(t)=r \cos \left(\omega_{1} t+\phi\right), \quad \dot{x}(t)=-r \omega_{1} \sin \left(\omega_{1} t+\phi\right),
$$

with an equation of constraint given by

$$
0=\dot{r} \cos \left[\omega_{1} t+\phi\right]-\dot{\phi} r \sin \left[\omega_{1} t+\phi\right] .
$$

We substitute Eq. (13), the derivatives of Eq. (13), and Eq. (14) into Eq. (12) and follow the method-of-averaging procedure to obtain the equations for $r$ and $\phi$ :

$$
\begin{aligned}
& \dot{r}=-\frac{\gamma r}{2}-\frac{\eta r^{3}}{8}-\frac{\lambda r \sin (\delta)}{4 \omega_{1}}, \\
& \dot{\phi}=\frac{3 \alpha r^{2}}{8 \omega_{1}}+\frac{\lambda \cos (\delta)}{4 \omega_{1}} .
\end{aligned}
$$

Setting Eq. (15) equal to zero yields the closed-loop amplitude-phase curve:

$$
r=\sqrt{-\frac{2 \lambda}{\omega_{1} \eta}\left(\sin (\delta)+\frac{2 \gamma \omega_{1}}{\lambda}\right)} .
$$

The vibration frequency is determined from the time rate of change of the phase, $\omega=\omega_{1}+d \phi / d t$ :

$$
\omega=\omega_{1}+\frac{\lambda}{4 \omega_{1}}\left(\cos (\delta)-\frac{3 \alpha}{\omega_{1} \eta} \sin (\delta)\right)-\frac{3 \alpha \gamma}{2 \omega_{1} \eta} .
$$

\section{Implementation and measurements}

Figure 5 depicts the implementation of a parametric phase-locked loop using our devices. We monitor the motion at $\omega$ using the piezoresistive readout. This signal is fed into the input of a Zurich Instruments (ZI) lock-in amplifier, where it is demodulated to extract the amplitude, $r$, and phase, $\phi$, with respect to the numerically controlled oscillator at $\omega$. The phase signal is fed into a PID loop, which adjusts the NCO frequency so as to minimize the phase error between $\phi$ and the desired phase condition, $\delta$. The NCO output is amplified and frequency doubled to provide the $2 \omega$ pump at the ZI output. There is no drive signal at $\omega$. Based on the convention in Eq. (12), the parametric resonator is phase locked to the pump when $\phi=-\delta / 2$. For the narrow-gap devices, application of a pump to a single adjacent electrode is sufficient to induce parametric oscillations, so both the capacitive and piezoresistive readouts can be used to monitor the resulting parametric oscillations and the phase from either readout can be used to establish the parametric PLL. For the wide-gap devices, both electrodes are required to induce parametric oscillations, so only the piezoresistive readout can be used for monitoring the motion and establishing a parametric PLL. The phase of the parametric pumps applied to the opposing electrodes should be the same, in order to double the parametric pump strength. 


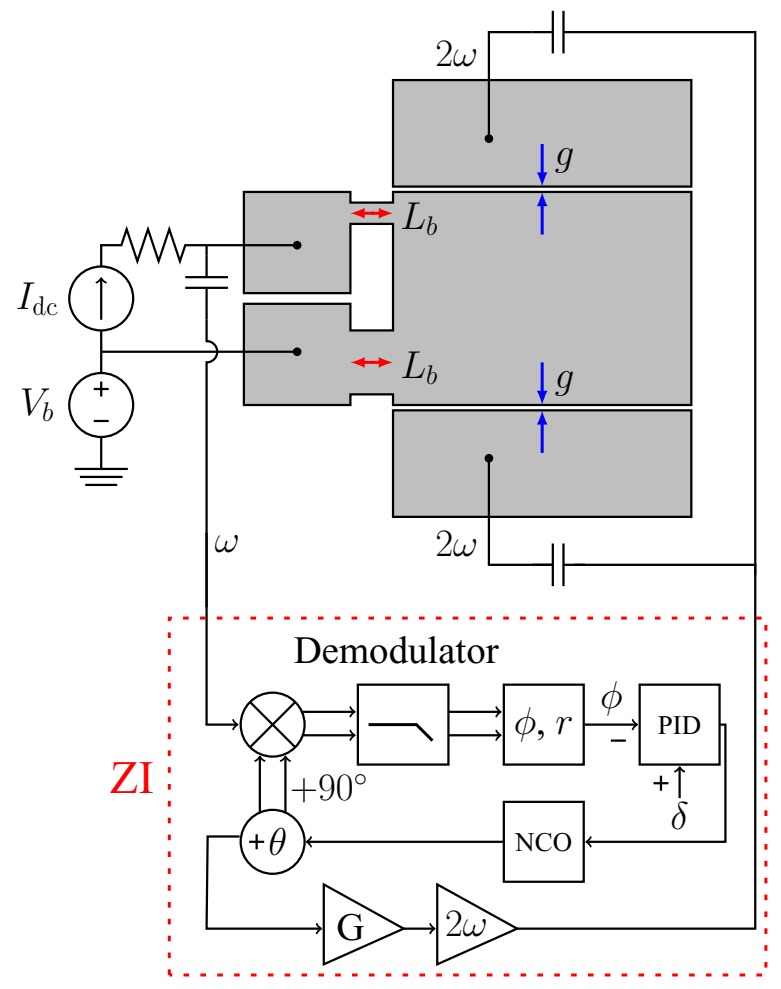

FIG. 5. Implementation of the parametric phase-locked loop using a nonlinearly damped resonator. Parametric phase control is possible for the resonators with sufficiently short support beams $\left(L_{b}\right)$ and wide gaps $(g)$.

Figure 6 depicts the measured stable and unstable branches of parametric resonators with $\eta>\eta_{0}$ using parametric phase control, confirming Eqs. (17) and (18). Starting at the largest pump amplitude and the smallest allowable vibration amplitude, we increase $\delta$ while measuring $r(\delta)$ and $\omega(\delta)$ to travel around the stable branch and onto the unstable branch (at which point the phase slope changes sign), then reverse the phase direction to return to the initial state. We repeat this procedure to measure successive $r(\delta)$ and $\omega(\delta)$ curves at progressively reduced pump amplitudes until the phase unlocks. Phase slipping occurs on the unstable branches in Fig. 6(b) when the phase slope switches from negative to positive, since the sign and magnitude of the proportional gain is optimized for operation on the stable branches. The model matches the amplitude and phase curves for both devices remarkably well once the static shift in the resonant frequency due to the parametric pump is accounted for [57] and the phase curves are shifted by a constant phase offset to account for the phase shift arising from the dc-decoupling capacitors and the reactance of the cables.

Phase control is possible if the phase slope of the stable branch is negative, as in Figs. 4(c) and 4(d). In our experiments, all phase-control attempts for devices with a positive phase slope are unsuccessful. The device immediately drops off the stable parametric resonance branch or the amplitude grows rapidly until the device shorts to an electrode. Analogous behavior in the vicinity of $\eta_{0}$ should be obtainable for parametric resonators with stiffening nonlinearity and negative nonlinear damping $[57,78]$.

\section{CONCLUSION AND OUTLOOK}

The phase-frequency curves of a parametric resonator can be easily measured by sweeping a parametric pump of sufficient amplitude across twice the resonant frequency. A negative phase slope implies that the nonlinear damping exceeds the critical value, so phase control can be used to operate on the unstable branches. Parametric phase control could be useful as a MEM or NEM timing reference,
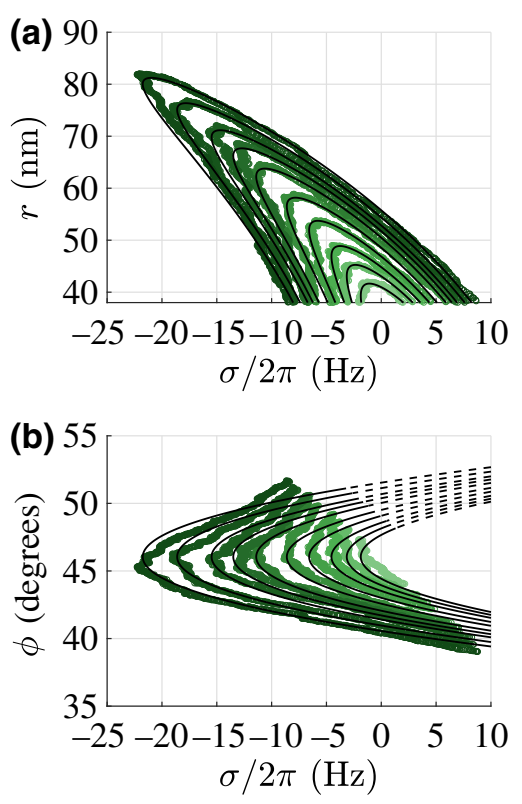
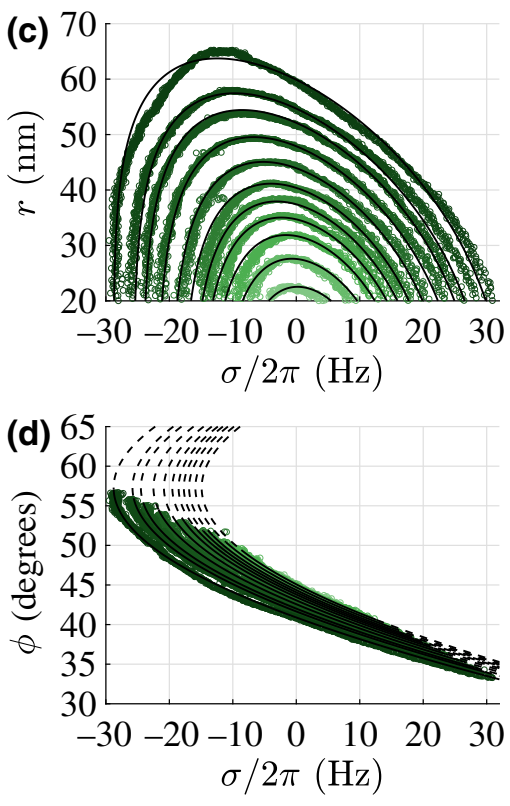

FIG. 6. Parametric phase-controlled measurements of the (a),(c) amplitude ( $r$ ) versus frequency detuning $(\sigma)$ and (b), (d) phase $(\phi)$ versus $\sigma$ for parametric oscillations of devices with wide gaps and short support beams, with nonlinear damping slightly above (a),(b) (device B) and well above (c),(d) (device C) the critical value, respectively. The solid (dashed) lines correspond to the nontrivial (trivial) solutions to the model in Eqs. (17) and (18). Note that $\phi=-\delta / 2$ is the independent variable and $r$ and $\sigma$ are the dependent variables in the measurements. 
provides a sensitive technique for extracting the nonlinear parameters of a mode, and enables further measurements of the dynamics of parametric resonators.

\section{ACKNOWLEDGMENTS}

Fabrication was performed in the nano@Stanford laboratories, which are supported by the National Science Foundation (NSF) as part of the National Nanotechnology Coordinated Infrastructure under Grant No. ECCS1542152, with support from the Defense Advanced Research Projects Agency Precise Robust Inertial Guidance for Munitions (PRIGM) Program, managed by Ron Polcawich and Robert Lutwak. J.M.L.M. is supported by the National Defense Science and Engineering Graduate (NDSEG) Fellowship and the E.K. Potter Stanford Graduate Fellowship. S.W.S. is supported by the NSF under Grants No. CMMI-1662619 and No. CMMI-1561829. We are grateful to Mark Dykman, Guillermo Villanueva, Daniel Rugar, and Marc Serra-Garcia for helpful discussions.

[1] J. A. Giordmaine and R. C. Miller, Tunable Coherent Parametric Oscillation in $\mathrm{LiNbO}_{3}$ at Optical Frequencies, Phys. Rev. Lett. 14, 973 (1965).

[2] S. Brosnan and R. Byer, Optical parametric oscillator threshold and linewidth studies, IEEE J. Quantum Electron. 15, 415 (1979).

[3] A. Dutt, K. Luke, S. Manipatruni, A. L. Gaeta, P. Nussenzveig, and M. Lipson, On-Chip Optical Squeezing, Phys. Rev. Appl. 3, 044005 (2015).

[4] B. Yurke, P. G. Kaminsky, R. E. Miller, E. A. Whittaker, A. D. Smith, A. H. Silver, and R. W. Simon, Observation of 4.2-K Equilibrium-Noise Squeezing via a JosephsonParametric Amplifier, Phys. Rev. Lett. 60, 764 (1988).

[5] C. M. Wilson, T. Duty, M. Sandberg, F. Persson, V. Shumeiko, and P. Delsing, Photon Generation in an Electromagnetic Cavity with a Time-Dependent Boundary, Phys. Rev. Lett. 105, 233907 (2010).

[6] M. Malnou, D. Palken, L. R. Vale, G. C. Hilton, and K. Lehnert, Optimal Operation of a Josephson Parametric Amplifier for Vacuum Squeezing, Phys. Rev. Appl. 9, 044023 (2018).

[7] D. Rugar and P. Grütter, Mechanical Parametric Amplification and Thermomechanical Noise Squeezing, Phys. Rev. Lett. 67, 699 (1991).

[8] K. L. Turner, S. A. Miller, P. G. Hartwell, N. C. MacDonald, S. H. Strogatz, and S. G. Adams, Five parametric resonances in a microelectromechanical system, Nature 396, 149 (1998).

[9] A. Dana, F. Ho, and Y. Yamamoto, Mechanical parametric amplification in piezoresistive gallium arsenide microcantilevers, Appl. Phys. Lett. 72, 1152 (1998).

[10] J. M. L. Miller, A. Ansari, D. B. Heinz, Y. Chen, I. B. Flader, D. D. Shin, L. G. Villanueva, and T. W. Kenny, Effective quality factor tuning mechanisms in micromechanical resonators, Appl. Phys. Rev. 5, 041307 (2018).
[11] M. Dykman, Fluctuating Nonlinear Oscillators: From Nanomechanics to Quantum Superconducting Circuits (Oxford University Press, Oxford, United Kingdom, 2012).

[12] H. B. Chan and C. Stambaugh, Activation Barrier Scaling and Crossover for Noise-Induced Switching in Micromechanical Parametric Oscillators, Phys. Rev. Lett. 99, 060601 (2007).

[13] H. B. Chan, M. I. Dykman, and C. Stambaugh, Paths of Fluctuation Induced Switching, Phys. Rev. Lett. 100, 130602 (2008).

[14] Z. R. Lin, Y. Nakamura, and M. I. Dykman, Critical fluctuations and the rates of interstate switching near the excitation threshold of a quantum parametric oscillator, Phys. Rev. E 92, 022105 (2015).

[15] J. F. Rhoads and S. W. Shaw, The impact of nonlinearity on degenerate parametric amplifiers, Appl. Phys. Lett. 96, 234101 (2010).

[16] A. Leuch, L. Papariello, O. Zilberberg, C. L. Degen, R. Chitra, and A. Eichler, Parametric Symmetry Breaking in a Nonlinear Resonator, Phys. Rev. Lett. 117, 214101 (2016).

[17] T. L. Heugel, M. Oscity, A. Eichler, O. Zilberberg, and R. Chitra, Classical many-body time crystals, arXiv:1903.02311 (2019).

[18] E. Goto, The parametron, a digital computing element which utilizes parametric oscillation, Proc. IRE 47, 1304 (1959).

[19] I. Mahboob and H. Yamaguchi, Bit storage and bit flip operations in an electromechanical oscillator, Nat. Nanotechnol. 3, 275 (2008).

[20] Z. R. Lin, K. Inomata, K. Koshino, W. Oliver, Y. Nakamura, J.-S. Tsai, and T. Yamamoto, Josephson parametric phaselocked oscillator and its application to dispersive readout of superconducting qubits, Nat. Commun. 5, 4480 (2014).

[21] I. Mahboob, H. Okamoto, and H. Yamaguchi, An electromechanical Ising Hamiltonian, Sci. Adv. 2, e1600236 (2016).

[22] Z. Nosan, P. Marki, N. Hauff, C. Knaut, and A. Eichler, Gate-controlled phase switching in a parametron, arXiv:1901.07764 (2019).

[23] M. Serra-Garcia, Turing-complete mechanical processor via automated nonlinear system design, Phys. Rev. E 100, 042202 (2019).

[24] L. D. Landau and E. M. Lifshitz, Mechanics (Pergamon, Oxford, United Kingdom, 1976), 3rd ed.

[25] M. Cartmell, Introduction to Linear, Parametric, and Nonlinear Vibrations (Chapman and Hall, London, United Kingdom, 1990).

[26] R. Lifshitz and M. C. Cross, in Nonlinear Dynamics of Nanosystems (Wiley, Weinheim, 2008), p. 221.

[27] A. H. Nayfeh and D. T. Mook, Nonlinear Oscillations (Wiley-VCH Verlag GmbH \& Co., Weinheim, Germany, 2008).

[28] R. B. Karabalin, R. Lifshitz, M. C. Cross, M. H. Matheny, S. Masmanidis, and M. L. Roukes, Signal Amplification by Sensitive Control of Bifurcation Topology, Phys. Rev. Lett. 106, 094102 (2011).

[29] L. Papariello, O. Zilberberg, A. Eichler, and R. Chitra, Ultrasensitive hysteretic force sensing with parametric nonlinear oscillators, Phys. Rev. E 94, 022201 (2016). 
[30] M. Sharma, E. H. Sarraf, R. Baskaran, and E. Cretu, Parametric resonance: Amplification and damping in MEMS gyroscopes, Sens. Actuators A 177, 79 (2012).

[31] C. H. Ahn, S. Nitzan, E. J. Ng, V. A. Hong, Y. Yang, T. Kimbrell, D. A. Horsley, and T. W. Kenny, Encapsulated high frequency $(235 \mathrm{kHz})$, high-Q $(100 \mathrm{k})$ disk resonator gyroscope with electrostatic parametric pump, Appl. Phys. Lett. 105, 243504 (2014).

[32] S. H. Nitzan, V. Zega, M. Li, C. H. Ahn, A. Corigliano, T. W. Kenny, and D. A. Horsley, Self-induced parametric amplification arising from nonlinear elastic coupling in a micromechanical resonating disk gyroscope, Sci. Rep. 5, 9036 (2015).

[33] J. Suh, M. D. LaHaye, P. M. Echternach, K. C. Schwab, and M. L. Roukes, Parametric amplification and back-action noise squeezing by a qubit-coupled nanoresonator, Nano Lett. 10, 3990 (2010).

[34] J. Gieseler, B. Deutsch, R. Quidant, and L. Novotny, Subkelvin Parametric Feedback Cooling of a Laser-Trapped Nanoparticle, Phys. Rev. Lett. 109, 103603 (2012).

[35] A. Vinante and P. Falferi, Feedback-Enhanced Parametric Squeezing of Mechanical Motion, Phys. Rev. Lett. 111, 207203 (2013).

[36] A. Pontin, M. Bonaldi, A. Borrielli, F. S. Cataliotti, F. Marino, G. A. Prodi, E. Serra, and F. Marin, Squeezing a Thermal Mechanical Oscillator by Stabilized Parametric Effect on the Optical Spring, Phys. Rev. Lett. 112, 023601 (2014).

[37] M. Poot, K. Fong, and H. Tang, Deep feedback-stabilized parametric squeezing in an opto-electromechanical system, New J. Phys. 17, 043056 (2015).

[38] E. J. Davis, Z. Wang, A. H. Safavi-Naeini, and M. H. Schleier-Smith, Painting Nonclassical States of Spin or Motion with Shaped Single Photons, Phys. Rev. Lett. 121, 123602 (2018).

[39] V. Zega, S. Nitzan, M. Li, C. Ahn, E. Ng, V. Hong, Y. Yang, T. Kenny, A. Corigliano, and D. Horsley, Predicting the closed-loop stability and oscillation amplitude of nonlinear parametrically amplified oscillators, Appl. Phys. Lett. 106, 233111 (2015).

[40] C. T. Nguyen, MEMS technology for timing and frequency control, IEEE Trans. Ultrason. Ferroelectr. Freq. Control 54, 251 (2007).

[41] S. Schmid, L. G. Villanueva, and M. L. Roukes, Fundamentals of Nanomechanical Resonators (Springer International Publishing AG Switzerland, Basel, Switzerland, 2016).

[42] M. Hossein-Zadeh, H. Rokhsari, A. Hajimiri, and K. J. Vahala, Characterization of a radiation-pressure-driven micromechanical oscillator, Phys. Rev. A 74, 023813 (2006).

[43] X. L. Feng, C. J. White, A. Hajimiri, and M. L. Roukes, A self-sustaining ultrahigh-frequency nanoelectromechanical oscillator, Nat. Nanotechnol. 3, 342 (2008).

[44] S. Zaliasl, J. C. Salvia, G. C. Hill, L. W. Chen, K. Joo, R. Palwai, N. Arumugam, M. Phadke, S. Mukherjee, H.C. Lee et al., A 3 ppm $1.5 \times 0.8 \mathrm{~mm}^{2} 1.0 \mu \mathrm{A} 32.768 \mathrm{kHz}$ MEMS-based oscillator, IEEE J. Solid-State Circuits 50, 291 (2015).

[45] C. Chen, D. H. Zanette, J. R. Guest, D. A. Czaplewski, and D. López, Self-Sustained Micromechanical Oscillator with Linear Feedback, Phys. Rev. Lett. 117, 017203 (2016).
[46] C.-C. Chu, S. Dey, T.-Y. Liu, C.-C. Chen, and S.-S. Li, Thermal-piezoresistive SOI-MEMS oscillators based on a fully differential mechanically coupled resonator array for mass sensing applications, J. Microelectromech. Syst. 27, 59 (2018).

[47] SiTime, Inc., Sunnyvale, CA (2019), https://www.sitime. $\mathrm{com} /$.

[48] K. L. Ekinci, Y. T. Yang, and M. L. Roukes, Ultimate limits to inertial mass sensing based upon nanoelectromechanical systems, J. Appl. Phys. 95, 2682 (2004).

[49] T. Kouh, O. Basarir, and K. Ekinci, Room-temperature operation of a nanoelectromechanical resonator embedded in a phase-locked loop, Appl. Phys. Lett. 87, 113112 (2005).

[50] L. G. Villanueva, R. B. Karabalin, M. H. Matheny, E. Kenig, M. C. Cross, and M. L. Roukes, A nanoscale parametric feedback oscillator, Nano Lett. 11, 5054 (2011).

[51] H. K. Lee, R. Melamud, S. Chandorkar, J. Salvia, S. Yoneoka, and T. W. Kenny, Stable operation of MEMS oscillators far above the critical vibration amplitude in the nonlinear regime, J. Microelectromech. Syst. 20, 1228 (2011).

[52] L. Villanueva, E. Kenig, R. Karabalin, M. Matheny, R. Lifshitz, M. Cross, and M. Roukes, Surpassing Fundamental Limits of Oscillators Using Nonlinear Resonators, Phys. Rev. Lett. 110, 177208 (2013).

[53] P. Taheri-Tehrani, M. Defoort, and D. Horsley, Synchronization of a micromechanical oscillator in different regimes of electromechanical nonlinearity, Appl. Phys. Lett. 111, 183503 (2017).

[54] M. Yuksel, E. Orhan, C. Yanik, A. B. Ari, A. Demir, and M. S. Hanay, Nonlinear nanomechanical mass spectrometry at the single-nanoparticle level, Nano Lett. 19, 3583 (2019).

[55] D. Senkal, E. Ng, V. Hong, Y. Yang, C. Ahn, T. Kenny, and A. Shkel, in 28th Int. Conf. Microelectromech. Syst. (IEEE MEMS conference, Estoril, Portugal, 2015), p. 29.

[56] J. Atalaya, T. W. Kenny, M. L. Roukes, and M. I. Dykman, Nonlinear damping and dephasing in nanomechanical systems, Phys. Rev. B 94, 195440 (2016).

[57] See the Supplemental Material at http://link.aps.org/supple mental/10.1103/PhysRevApplied.12.044053 for additional modeling and measurement details.

[58] M. V. Requa and K. L. Turner, Electromechanically driven and sensed parametric resonance in silicon microcantilevers, Appl. Phys. Lett. 88, 263508 (2006).

[59] R. P. Feynman, R. B. Leighton, and M. Sands, The Feynman Lectures on Physics, Vol. I: The New Millennium Edition: Mainly Mechanics, Radiation, and Heat (Basic Books, New York, New York, 2011).

[60] A. Kraus, A. Erbe, R. H. Blick, G. Corso, and K. Richter, Parametric frequency tuning of phase-locked nanoelectromechanical resonators, Appl. Phys. Lett. 79, 3521 (2001).

[61] W. Zhang, R. Baskaran, and K. L. Turner, Effect of cubic nonlinearity on auto-parametrically amplified resonant MEMS mass sensor, Sens. Actuators A 102, 139 (2002).

[62] B. E. DeMartini, J. F. Rhoads, K. L. Turner, S. W. Shaw, and J. Moehlis, Linear and nonlinear tuning of parametrically excited MEMS oscillators, J. Microelectromech. Syst. 16, 310 (2007). 
[63] P. Prasad, N. Arora, and A. K. Naik, Parametric amplification in $\mathrm{MoS}_{2}$ drum resonator, Nanoscale 9, 18299 (2017).

[64] R. J. Dolleman, S. Houri, A. Chandrashekar, F. Alijani, H. S. van der Zant, and P. G. Steeneken, Opto-thermally excited multimode parametric resonance in graphene membranes, Sci. Rep. 8, 9366 (2018).

[65] P. G. Steeneken, K. Le Phan, M. J. Goossens, G. E. J. Koops, G. J. A. M. Brom, C. van der Avoort, and J. T. M. van Beek, Piezoresistive heat engine and refrigerator, Nat. Phys. 7, 354 (2011).

[66] J. M. L. Miller, H. Zhu, D. B. Heinz, Y. Chen, I. B. Flader, D. D. Shin, J. E.-Y. Lee, and T. W. Kenny, ThermalPiezoresistive Tuning of the Effective Quality Factor of a Micromechanical Resonator, Phys. Rev. Appl. 10, 044055 (2018).

[67] Y. Yang, E. J. Ng, Y. Chen, I. B. Flader, and T. W. Kenny, A unified epi-seal process for fabrication of highstability microelectromechanical devices, J. Microelectromech. Syst. 25, 489 (2016).

[68] M. Agarwal, S. A. Chandorkar, H. Mehta, R. N. Candler, B. Kim, M. A. Hopcroft, R. Melamud, C. M. Jha, G. Bahl, G. Yama et al., A study of electrostatic force nonlinearities in resonant microstructures, Appl. Phys. Lett. 92, 104106 (2008).

[69] S. Zaitsev, O. Shtempluck, E. Buks, and O. Gottlieb, Nonlinear damping in a micromechanical oscillator, Nonlinear Dyn. 67, 859 (2012).

[70] M. Imboden, O. Williams, and P. Mohanty, Nonlinear dissipation in diamond nanoelectromechanical resonators, Appl. Phys. Lett. 102, 103502 (2013).
[71] I. Mahboob, N. Perrissin, K. Nishiguchi, D. Hatanaka, Y. Okazaki, A. Fujiwara, and H. Yamaguchi, Dispersive and dissipative coupling in a micromechanical resonator embedded with a nanomechanical resonator, Nano Lett. 15, 2312 (2015).

[72] P. M. Polunin, Y. Yang, M. I. Dykman, T. W. Kenny, and S. W. Shaw, Characterization of MEMS resonator nonlinearities using the ringdown response, J. Microelectromech. Syst. 25, 297 (2016).

[73] A. Eichler, J. Moser, J. Chaste, M. Zdrojek, I. Wilson-Rae, and A. Bachtold, Nonlinear damping in mechanical resonators made from carbon nanotubes and graphene, Nat. Nanotechnol. 6, 339 (2011).

[74] A. Croy, D. Midtvedt, A. Isacsson, and J. M. Kinaret, Nonlinear damping in graphene resonators, Phys. Rev. B 86, 235435 (2012).

[75] T. Miao, S. Yeom, P. Wang, B. Standley, and M. Bockrath, Graphene nanoelectromechanical systems as stochasticfrequency oscillators, Nano Lett. 14, 2982 (2014).

[76] J. Güttinger, A. Noury, P. Weber, A. M. Eriksson, C. Lagoin, J. Moser, C. Eichler, A. Wallraff, A. Isacsson, and A. Bachtold, Energy-dependent path of dissipation in nanomechanical resonators, Nat. Nanotechnol. 12, 631 (2017).

[77] B. D. Hauer, C. Doolin, K. S. D. Beach, and J. P. Davis, A general procedure for thermomechanical calibration of nano/micro-mechanical resonators, Ann. Phys. 339, 181 (2013).

[78] X. Dong, M. I. Dykman, and H. B. Chan, Strong negative nonlinear friction from induced two-phonon processes in vibrational systems, Nat. Commun. 9, 3241 (2018). 